\title{
On Tornheim's double series
}

\author{
by
}

James G. Huard (Buffalo, N.Y.), Kenneth S. Williams (Ottawa, Ont.) and Zhang NAN-Yue (Beijing)

1. Introduction. We call the double infinite series

$$
T(r, s, t)=\sum_{\mu, \nu=1}^{\infty} \frac{1}{\mu^{r} \nu^{s}(\mu+\nu)^{t}} \quad(r, s, t \text { nonnegative integers })
$$

a Tornheim series, after Tornheim who made a systematic and thorough investigation of this interesting series in a paper [6] published in 1950. The Tornheim series $T(r, 0, t)$, when rewritten in the form

$$
T(r, 0, t)=\sum_{\tau=1}^{\infty} \frac{1}{(\tau+1)^{t}} \sum_{\mu=1}^{\tau} \frac{1}{\mu^{r}},
$$

arises in the study of the gamma function (see, for example, [3, p. 47]). The series $T(r, s, t)$ is finite if and only if

$$
r+t>1, \quad s+t>1, \quad \text { and } \quad r+s+t>2 .
$$

The basic properties of $T(r, s, t)$ (assuming (1.1)) are the following

(1.2) $T(r, s, t)=T(s, r, t)$,

(1.3) $\quad T(r, s, 0)=\zeta(r) \zeta(s)$,

(1.4) $\quad T(r, 0, t)+T(t, 0, r)=\zeta(r) \zeta(t)-\zeta(r+t), \quad r \geq 2$,

(1.5) $T(r, s-1, t+1)+T(r-1, s, t+1)=T(r, s, t), \quad r \geq 1, s \geq 1$,

where $\zeta$ denotes the Riemann zeta function.

1991 Mathematics Subject Classification: Primary 11M06; Secondary 30B99, 33E20, 40A05, 40B05.

Key words and phrases: Tornheim's double series, Riemann zeta function.

Research of the second author supported by Natural Sciences and Engineering Research Council of Canada grant A-7233.

Research of the third author supported by the National Natural Sciences Foundation of China. 
Properties (1.3) and (1.4) give us a glimpse of the fascinating interplay between Tornheim series and the Riemann zeta function which we explore in this paper. The key to understanding this interplay is the recurrence relation (1.5). For a fixed value $N(\geq 3)$ of $r+s+t$, we place the (finite) $T(r, s, t)$ in a hexagonal array $\mathcal{T}_{N}$ of $N$ rows by arranging those $T(r, s, t)$ with $t=k$ in the $(N-k)$ th row in order of increasing $s$. For example, the array $\mathcal{T}_{5}$ is

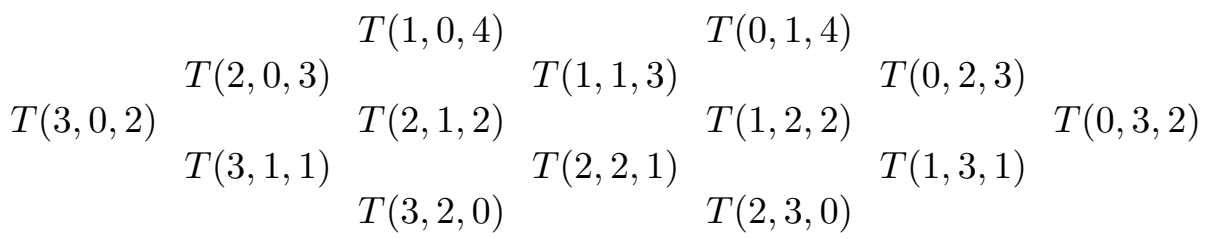

The Tornheim hexagon $\mathcal{T}_{N}$ is symmetric about its vertical axis in view of (1.2). The recurrence relation (1.5) shows that each entry with $r \neq 0$ and $s \neq 0$ is the sum of the two entries immediately above it, as in Pascal's triangle. Applying this relation successively, we see that every entry can be expressed as a linear combination of those entries with $r=0$ or $s=0$, and thus (in view of the symmetry) just in terms of those with $s=0$. For example, in $\mathcal{T}_{5}$,

$$
\begin{aligned}
T(2,2,1) & =T(2,1,2)+T(1,2,2) \\
& =T(2,0,3)+2 T(1,1,3)+T(0,2,3) \\
& =T(2,0,3)+2 T(1,0,4)+2 T(0,1,4)+T(0,2,3) \\
& =4 T(1,0,4)+2 T(2,0,3) .
\end{aligned}
$$

In general, if $r$ and $s$ are integers satisfying

$$
0 \leq r \leq N-2, \quad 0 \leq s \leq N-2, \quad 1 \leq r+s \leq N,
$$

then

$$
\begin{aligned}
& T(r, s, N-r-s) \\
& =\sum_{i=1}^{r}\left(\begin{array}{c}
r+s-i-1 \\
r-i
\end{array}\right) T_{i}+\sum_{i=1}^{s}\left(\begin{array}{c}
r+s-i-1 \\
s-i
\end{array}\right) T_{i},
\end{aligned}
$$

where

$$
T_{i}=T(i, 0, N-i), \quad i=1,2, \ldots, N-2,
$$

which is easily established by induction. We note that $T_{N-1}$ is not finite. From (1.4) we see that

$$
T_{i}+T_{N-i}=\zeta(i) \zeta(N-i)-\zeta(N), \quad i=2, \ldots, N-2 .
$$


When $r+s=N$ the left side of (1.6) is given by (1.3), and we obtain the following system of $[N / 2]-1$ linear equations for the $T_{i}$ :

$$
\begin{aligned}
\sum_{i=1}^{r}\left(\begin{array}{c}
N-i-1 \\
r-i
\end{array}\right) T_{i}+\sum_{i=1}^{N-r}\left(\begin{array}{c}
N-i-1 \\
N-r-i
\end{array}\right) T_{i}=\zeta(r) \zeta(N-r) \\
r=2,3, \ldots,[N / 2]
\end{aligned}
$$

Using (1.7) to replace $T_{i}$ by $\zeta(i) \zeta(N-i)-\zeta(N)-T_{N-i}$ for $i=[N / 2]+1$, $\ldots, N-2$, and using the value

$$
T_{1}=\frac{1}{2}(N-1) \zeta(N)-\frac{1}{2} \sum_{j=2}^{N-2} \zeta(j) \zeta(N-j)
$$

found by Tornheim [6, eq. (10)], we obtain from (1.8) a system $\left(S_{N}\right)$ of $[N / 2]-1$ linear equations for the $[N / 2]-1$ quantities $T_{2}, \ldots, T_{[N / 2]}$.

In addition to (1.9), Tornheim has evaluated $T(r, s, t)$ explicitly in certain other cases. For example he has shown for $N \geq 3$ that

$$
\begin{gathered}
T(0,0, N)=\zeta(N-1)-\zeta(N), \\
T(1, N-2,1)=\frac{1}{2}(N+1) \zeta(N)-\frac{1}{2} \sum_{j=2}^{N-2} \zeta(j) \zeta(N-j), \\
T(1,1, N-2)=(N-1) \zeta(N)-\sum_{j=2}^{N-2} \zeta(j) \zeta(N-j) .
\end{gathered}
$$

Further, when $N=r+s+t$ is odd, he proved that $T(r, s, t)$ is a polynomial in $\zeta(2), \ldots, \zeta(N)$ with rational coefficients but did not give the polynomial.

The purpose of this paper is to determine $T(r, s, t)$ explicitly when $N=$ $r+s+t$ is odd. We show that $T(r, s, t)$ is an integral linear combination of the products $\zeta(2 i) \zeta(N-2 i), i=0,1, \ldots,(N-3) / 2$, except when $r=s=0$. We also indicate why our approach does not appear to work when $r+s+t$ is even. We use the identity

$$
\begin{aligned}
\frac{(-1)^{v}}{k^{v}(k+r)^{u}}= & \sum_{j=1}^{v}\left(\begin{array}{c}
u+v-j-1 \\
v-j
\end{array}\right) \frac{(-1)^{j}}{r^{u+v-j} k^{j}} \\
& +\sum_{j=1}^{u}\left(\begin{array}{c}
u+v-j-1 \\
u-j
\end{array}\right) \frac{1}{r^{u+v-j}(k+r)^{j}}
\end{aligned}
$$

where $k$ and $r$ are positive integers, and $u$ and $v$ are nonnegative integers not both zero, which follows easily from $[3$, p. 48 , eq. (9)]. When $N$ is odd 
we use (1.12) in Section 3 to show that the system $\left(S_{N}\right)$ uniquely determines $T_{2}, \ldots, T_{[N / 2]}$. We prove

TheOREm 1. Let $N$ be an odd integer with $N \geq 3$. Then, for $i=$ $1, \ldots, N-2$, we have

$$
\begin{aligned}
T(i, 0, N-i)= & (-1)^{i} \sum_{j=0}^{[(N-i-1) / 2]}\left(\begin{array}{c}
N-2 j-1 \\
i-1
\end{array}\right) \zeta(2 j) \zeta(N-2 j) \\
& +(-1)^{i} \sum_{j=0}^{[i / 2]}\left(\begin{array}{c}
N-2 j-1 \\
N-i-1
\end{array}\right) \zeta(2 j) \zeta(N-2 j)+\zeta(0) \zeta(N),
\end{aligned}
$$

where we recall that $\zeta(0)=-1 / 2$.

However, when $N$ is even the system $\left(S_{N}\right)$ is not in general sufficient to find all of the $T_{i}$, for example when $N=8,10,12,14,16,18$.

The value of a general $T(r, s, t)$ when $r+s+t$ is odd is obtained in Section 4 by using in (1.6) the values of the $T_{i}$ given in Theorem 1 and appealing to a combinatorial identity (Lemma 2.1) to evaluate the resulting sums of binomial coefficients. We prove

Theorem 2. Let $N$ be an odd integer with $N \geq 3$. Let $r$ and $s$ be nonnegative integers satisfying $1 \leq r+s \leq N-1, r \leq N-2, s \leq N-2$. Set

$$
\begin{aligned}
E_{N}(r, s)= & (-1)^{r} \sum_{i=0}^{[(N-r-s-1) / 2]}\left(\begin{array}{c}
N-2 i-s-1 \\
r-1
\end{array}\right) \zeta(2 i) \zeta(N-2 i) \\
& +(-1)^{r} \sum_{i=0}^{[r / 2]}\left(\begin{array}{c}
N-2 i-s-1 \\
N-r-s-1
\end{array}\right) \zeta(2 i) \zeta(N-2 i) .
\end{aligned}
$$

Then

$$
T(r, s, N-r-s)=E_{N}(r, s)+E_{N}(s, r) .
$$

Theorem 2 covers all nonnegative integers $r, s, t$ for which $T(r, s, t)$ is finite and $r+s+t=N$ is odd except for the case $r=s=0$, which is covered by (1.10), and the case $t=0$, which is covered by (1.3). All of Tornheim's other evaluations [6] are special cases of the above theorem. Furthermore, taking $r=s=t=2 k+1(k \geq 0)$ in Theorem 2, we deduce the result

$$
\begin{array}{r}
T(2 k+1,2 k+1,2 k+1) \\
=-4 \sum_{i=0}^{k}\left(\begin{array}{c}
4 k-2 i+1 \\
2 k
\end{array}\right) \zeta(2 i) \zeta(6 k-2 i+3) .
\end{array}
$$


The problem of evaluating $T(2 k+1,2 k+1,2 k+1)$ was posed in 1958 by Mordell [2], who showed that $T(2 k, 2 k, 2 k)$ is a rational multiple of $\pi^{6 k}$ for $k \geq 1$, but gave the multiple only for $k=1$. In 1985 Subbarao and Sitaramachandrarao [5] gave the explicit evaluation (with a misprint corrected)

$$
T(2 k, 2 k, 2 k)=\frac{8 k}{3((2 k) !)^{2}} \sum_{i=0}^{k}\left(\begin{array}{c}
2 k \\
2 i
\end{array}\right)(2 i) !(4 k-2 i-1) ! \zeta(2 i) \zeta(6 k-2 i),
$$

which can be rewritten in the simpler form

$$
T(2 k, 2 k, 2 k)=\frac{4}{3} \sum_{i=0}^{k}\left(\begin{array}{c}
4 k-2 i-1 \\
2 k-1
\end{array}\right) \zeta(2 i) \zeta(6 k-2 i),
$$

and then combined with (1.14) to give

TheOREM 3. For $r \geq 1$,

$$
T(r, r, r)=\frac{4}{1+2(-1)^{r}} \sum_{i=0}^{[r / 2]}\left(\begin{array}{c}
2 r-2 i-1 \\
r-1
\end{array}\right) \zeta(2 i) \zeta(3 r-2 i) .
$$

2. A combinatorial identity. As usual the binomial coefficient $\left(\begin{array}{l}x \\ n\end{array}\right)$ is defined for any real number $x$ and any integer $n$. We just note the properties

$$
\left(\begin{array}{c}
-x \\
n
\end{array}\right)=(-1)^{n}\left(\begin{array}{c}
x+n-1 \\
n
\end{array}\right)
$$

and, for any integer $m$,

$$
\left(\begin{array}{c}
m \\
n
\end{array}\right)=0 \text { if and only if } n<0 \text { or } 0 \leq m<n .
$$

The following identity is used in the proof of Theorem 2 .

Lemma 2.1. For nonnegative integers $m, n, p$ and $q$ with $p \geq q$, we have

$$
\sum_{k=0}^{m}(-1)^{k}\left(\begin{array}{c}
q \\
p-k
\end{array}\right)\left(\begin{array}{c}
m+n-k \\
n
\end{array}\right)=(-1)^{m}\left(\begin{array}{c}
q-n-1 \\
m+q-p
\end{array}\right) .
$$

Proof. We have

$$
\begin{aligned}
& \sum_{k=0}^{m}(-1)^{k}\left(\begin{array}{c}
q \\
p-k
\end{array}\right)\left(\begin{array}{c}
m+n-k \\
n
\end{array}\right)(-1)^{m} \sum_{k=0}^{m}(-1)^{k}\left(\begin{array}{c}
q \\
p-m+k
\end{array}\right)\left(\begin{array}{c}
n+k \\
n
\end{array}\right) \\
&=(-1)^{m} \sum_{k=0}^{m-p+q}(-1)^{k}\left(\begin{array}{c}
q \\
p-m+k
\end{array}\right)\left(\begin{array}{c}
n+k \\
k
\end{array}\right)
\end{aligned}
$$




$$
\begin{aligned}
& =(-1)^{m} \sum_{k=0}^{m-p+q}\left(\begin{array}{c}
q \\
m-p+q-k
\end{array}\right)\left(\begin{array}{c}
-n-1 \\
k
\end{array}\right) \quad \text { (by (2.1)) } \\
& =(-1)^{m}\left(\begin{array}{c}
q-n-1 \\
m-p+q
\end{array}\right)
\end{aligned}
$$

by the Vandermonde convolution (see $[1$, p. 22] or $[4$, p. 8]).

3. Evaluation of $T(i, 0, N-i), N$ (odd) $\geq 3$. We begin this section with two recurrence relations involving the $T_{j}$. Each of these is obtained by summing the identity (1.12) and making use of (1.7) and (1.8).

\section{Then}

Proposition 3.1. Let $N$ be an integer with $N \geq 3$, and let $i=1, \ldots, N-2$.

(a) $\quad\left(1-(-1)^{i}\right) T_{i}+\sum_{j=1}^{i-1}\left(\begin{array}{c}N-j-1 \\ N-i-1\end{array}\right) T_{j}$

$$
\begin{aligned}
= & -\sum_{j=i+1}^{N-2}\left(\begin{array}{c}
j-1 \\
i-1
\end{array}\right) \zeta(j) \zeta(N-j) \\
& -\sum_{j=2}^{i}(-1)^{j}\left(\begin{array}{c}
N-j-1 \\
N-i-1
\end{array}\right) \zeta(j) \zeta(N-j)+\left(\begin{array}{c}
N-1 \\
i
\end{array}\right) \zeta(N) .
\end{aligned}
$$

(b) $\quad\left((-1)^{N}-(-1)^{i}\right) T_{i}+(-1)^{N} \sum_{j=1}^{i-1}\left(\begin{array}{c}N-j-1 \\ N-i-1\end{array}\right) T_{j}$

$$
\begin{aligned}
= & \sum_{j=i+1}^{N-2}(-1)^{j}\left(\begin{array}{c}
j-1 \\
i-1
\end{array}\right) \zeta(j) \zeta(N-j) \\
& +(-1)^{N} \sum_{j=2}^{i}\left(\begin{array}{c}
N-j-1 \\
N-i-1
\end{array}\right) \zeta(j) \zeta(N-j) \\
& +\left((-1)^{i}-(-1)^{N}\left(\begin{array}{c}
N-1 \\
i-1
\end{array}\right)\right) \zeta(N) .
\end{aligned}
$$

Proof. When $i=1$, (a) holds by (1.9), and (b) follows from

$$
\begin{aligned}
\sum_{j=2}^{N-2}(-1)^{j} \zeta(j) \zeta(N-j) & \left.=\sum_{j=2}^{N-2}(-1)^{j} T(j, N-j, 0) \quad \text { (by }(1.3)\right) \\
& =\sum_{j=2}^{N-2}(-1)^{j}\{T(j, N-j-1,1)+T(j-1, N-j, 1)\}
\end{aligned}
$$




$$
\begin{aligned}
& =(-1)^{N-2} T(N-2,1,1)+T(1, N-2,1) \\
& =\left(1+(-1)^{N}\right) T(1, N-2,1) \quad(\text { by }(1.2)) \\
& =\left(1+(-1)^{N}\right)\left\{T_{1}+\zeta(N)\right\} \quad(\text { by }(1.9) \text { and }(1.11)) .
\end{aligned}
$$

Thus we may assume that $2 \leq i \leq N-2$.

(a) Collecting together the terms with $j=1$ in (1.12) (with $v=i$, $u=N-i)$, we have

$$
\begin{aligned}
\frac{(-1)^{i}}{k^{i}(k+r)^{N-i}}= & \sum_{j=2}^{i}\left(\begin{array}{c}
N-j-1 \\
i-j
\end{array}\right) \frac{(-1)^{j}}{r^{N-j} k^{j}} \\
& +\sum_{j=2}^{N-i}\left(\begin{array}{c}
N-j-1 \\
N-i-j
\end{array}\right) \frac{1}{r^{N-j}(k+r)^{j}} \\
& +\left(\begin{array}{c}
N-2 \\
i-1
\end{array}\right) \frac{1}{r^{N-1}}\left(\frac{1}{k+r}-\frac{1}{k}\right) .
\end{aligned}
$$

Summing over all positive integers $k$ and $r$, we obtain

$$
\begin{aligned}
(-1)^{i} T_{i}= & \sum_{j=2}^{i}(-1)^{j}\left(\begin{array}{c}
N-j-1 \\
i-j
\end{array}\right) \zeta(j) \zeta(N-j)+\sum_{j=2}^{N-i}\left(\begin{array}{c}
N-j-1 \\
N-i-j
\end{array}\right) T_{N-j} \\
& -\left(\begin{array}{c}
N-2 \\
i-1
\end{array}\right) T(1, N-2,1) .
\end{aligned}
$$

Hence

$$
\begin{aligned}
& (-1)^{i} T_{i}=\sum_{j=2}^{N-i}\left(\begin{array}{c}
N-j-1 \\
N-i-j
\end{array}\right)\left(\zeta(j) \zeta(N-j)-\zeta(N)-T_{j}\right) \\
& \quad+\sum_{j=2}^{i}(-1)^{j}\left(\begin{array}{c}
N-j-1 \\
N-i-1
\end{array}\right) \zeta(j) \zeta(N-j)-\left(\begin{array}{c}
N-2 \\
i-1
\end{array}\right)\left\{T_{1}+\zeta(N)\right\}
\end{aligned}
$$

by (1.7), (1.9) and (1.11). Appealing to (1.8), and noting that

$$
\sum_{j=2}^{N-i}\left(\begin{array}{c}
N-j-1 \\
N-i-j
\end{array}\right)=\left(\begin{array}{c}
N-2 \\
i
\end{array}\right),
$$

we deduce that

$$
\begin{aligned}
(-1)^{i} T_{i}= & \sum_{j=i}^{N-2}\left(\begin{array}{c}
j-1 \\
i-1
\end{array}\right) \zeta(j) \zeta(N-j)-\left(\left(\begin{array}{c}
N-2 \\
i
\end{array}\right)+\left(\begin{array}{c}
N-2 \\
i-1
\end{array}\right)\right) \zeta(N) \\
& -\zeta(i) \zeta(N-i)+\sum_{j=1}^{i}\left(\begin{array}{c}
N-j-1 \\
i-j
\end{array}\right) T_{j}
\end{aligned}
$$




$$
+\sum_{j=2}^{i}(-1)^{j}\left(\begin{array}{c}
N-j-1 \\
N-i-1
\end{array}\right) \zeta(j) \zeta(N-j),
$$

from which (a) follows.

(b) Replacing $i$ by $N-i$ in (3.1), multiplying by $(-1)^{N}$, and appealing to (1.7), we find that

$$
\begin{aligned}
(-1)^{i} \zeta(i) \zeta(N-i) & -(-1)^{i} \zeta(N)-(-1)^{i} T_{i} \\
= & (-1)^{N} \sum_{j=2}^{i}\left(\begin{array}{c}
N-j-1 \\
N-i-1
\end{array}\right)\left(\zeta(j) \zeta(N-j)-\zeta(N)-T_{j}\right) \\
& +(-1)^{N} \sum_{j=2}^{N-i}(-1)^{j}\left(\begin{array}{c}
N-j-1 \\
i-1
\end{array}\right) \zeta(j) \zeta(N-j) \\
& -(-1)^{N}\left(\begin{array}{c}
N-2 \\
N-i-1
\end{array}\right)\left(T_{1}+\zeta(N)\right) .
\end{aligned}
$$

Equation (b) now follows after rearranging, noting that $\sum_{j=1}^{i}\left(\begin{array}{l}N-j-1 \\ N-i-1\end{array}\right)=$ $\left(\begin{array}{c}N-1 \\ i-1\end{array}\right)$, and changing $j$ to $N-j$ in the second summation.

Proof of Theorem 1. As $N$ is odd, adding equations (a) and (b) of Proposition 3.1 eliminates the term $\sum_{j=1}^{i-1}\left(\begin{array}{c}N-j-1 \\ i-j\end{array}\right) T_{j}$, and we obtain

$$
\begin{aligned}
-2(-1)^{i} T_{i}= & -2 \sum_{\substack{j=i+1 \\
j \text { odd }}}^{N-2}\left(\begin{array}{c}
j-1 \\
i-1
\end{array}\right) \zeta(j) \zeta(N-j) \\
& -2 \sum_{\substack{j=2 \\
j \text { even }}}^{i}\left(\begin{array}{c}
N-j-1 \\
N-i-1
\end{array}\right) \zeta(j) \zeta(N-j) \\
& -2\left\{\left(\begin{array}{c}
N-1 \\
N-i-1
\end{array}\right)+\left(\begin{array}{c}
N-1 \\
i-1
\end{array}\right)+(-1)^{i}\right\} \zeta(0) \zeta(N) \\
= & -2 \sum_{k=0}^{[(N-i-1) / 2]}\left(\begin{array}{c}
N-2 k-1 \\
i-1
\end{array}\right) \zeta(2 k) \zeta(N-2 k) \\
& -2 \sum_{k=0}^{[i / 2]}\left(\begin{array}{c}
N-2 k-1 \\
N-i-1
\end{array}\right) \zeta(2 k) \zeta(N-2 k)-2(-1)^{i} \zeta(0) \zeta(N),
\end{aligned}
$$

from which Theorem 1 follows.

When $N$ is even, the left sides of (a) and (b) of Proposition 3.1 are the same, and equating their right sides, we obtain the following result. 
Proposition 3.2. Let $N$ be an even integer with $N \geq 4$, and let $i=$ $1, \ldots, N / 2$. Then

$$
\begin{array}{r}
\sum_{j=[i / 2]+1}^{(N-2) / 2}\left(\begin{array}{c}
2 j-1 \\
i-1
\end{array}\right) \zeta(2 j) \zeta(N-2 j)+\sum_{j=1}^{[i / 2]}\left(\begin{array}{c}
N-2 j-1 \\
N-i-1
\end{array}\right) \zeta(2 j) \zeta(N-2 j) \\
=\frac{1}{2}\left\{\left(\begin{array}{c}
N \\
i
\end{array}\right)-(-1)^{i}\right\} \zeta(N) .
\end{array}
$$

We remark that when $i=1$ Proposition 3.2 is a well-known identity (see for example [6, p. 308]).

We now discuss briefly the case when $N(\geq 4)$ is even. Recall that $\left(S_{N}\right)$ is a system of $[N / 2]-1$ linear equations in the $[N / 2]-1$ quantities $T_{i}$ $(i=2, \ldots,[N / 2])$. When $N$ is even, (1.7) gives

$$
T_{N / 2}=\frac{1}{2} \zeta(N / 2)^{2}-\frac{1}{2} \zeta(N)
$$

Using this in $\left(S_{N}\right)$, we obtain a new system $\left(S_{N}^{\prime}\right)$ of $N / 2-1$ linear equations in the $N / 2-2$ quantities $T_{i}(i=2, \ldots, N / 2-1)$. The system $\left(S_{4}^{\prime}\right)$ is trivial. When $N=6,8, \ldots, 18$ the rank of the system $\left(S_{N}^{\prime}\right)$ is $[N / 3]-1$. Thus $\left(S_{N}^{\prime}\right)$ does not determine all of the $T_{i}$ when $N=8,10, \ldots, 18$, but does when $N=6$. It would be of interest to know if the rank is given by $[N / 3]-1$ for $N \geq 20$.

When $N=4$, Proposition 3.2 gives the relation $2 \zeta(2)^{2}=5 \zeta(4)$, which we use to obtain $T(1,0,3)=\frac{1}{4} \zeta(4)$ and $T(2,0,2)=\frac{3}{4} \zeta(4)$. When $N=6$, the same proposition gives $4 \zeta(2) \zeta(4)=7 \zeta(6)$, and hence

$$
\begin{array}{ll}
T(1,0,5)=-\frac{1}{2} \zeta(3)^{2}+\frac{3}{4} \zeta(6), & T(2,0,4)=\zeta(3)^{2}-\frac{4}{3} \zeta(6), \\
T(3,0,3)=\frac{1}{2} \zeta(3)^{2}-\frac{1}{2} \zeta(6), & T(4,0,2)=-\zeta(3)^{2}+\frac{25}{12} \zeta(6) .
\end{array}
$$

When $N=8$, after a little algebra, we have $3 \zeta(2) \zeta(6)=5 \zeta(8)$, and $6 \zeta(4)^{2}=$ $7 \zeta(8)$. The system $\left(S_{8}^{\prime}\right)$ reduces to the single equation

$$
5 T(2,0,6)+2 T(3,0,5)=10 \zeta(3) \zeta(5)-\frac{119}{8} \zeta(8),
$$

so we are unable to determine the values of $T(2,0,6)$ and $T(3,0,5)$. When $N=10$, the system $\left(S_{10}^{\prime}\right)$ determines only the quantities $7 T(2,0,8)+$ $2 T(3,0,7)$ and $T(3,0,7)+T(4,0,6)$.

4. Evaluation of $T(r, s, N-r-s)$ for $N$ (odd) $\geq 3$ : Proof of Theorem 2. Let $N$ be an odd integer with $N \geq 3$, and let $r$ and $s$ be nonnegative integers satisfying $1 \leq r+s \leq N-1, r \leq N-2, s \leq N-2$. When $r=0$ or $s=0$ Theorem 2 follows from Theorem 1 and (1.2). Thus we have only to prove Theorem 2 when $r$ and $s$ are positive integers. From 
(1.6) we may write

$$
T(r, s, N-r-s)=U_{N}(r, s)+U_{N}(s, r),
$$

where

$$
U_{N}(r, s)=\sum_{i=1}^{r}\left(\begin{array}{c}
r+s-i-1 \\
s-1
\end{array}\right) T_{i} .
$$

In (1.6) the range of summation of each of the two sums may be extended up to $j=(N-3) / 2$ provided that the term $\zeta(i) \zeta(N-i)$ is appended when $i$ is odd and at least 3 , since in this case there is a contribution from the term with $j=(N-i) / 2$. We therefore obtain

$$
U_{N}(r, s)=S_{1}(r, s)+S_{2}(r, s)+S_{3}(r, s)+S_{4}(r, s),
$$

where

$$
\begin{aligned}
S_{1}(r, s) & =\sum_{j=0}^{(N-3) / 2} \zeta(2 j) \zeta(N-2 j) \sum_{i=1}^{r}(-1)^{i}\left(\begin{array}{c}
r+s-i-1 \\
s-1
\end{array}\right)\left(\begin{array}{c}
N-2 j-1 \\
i-1
\end{array}\right), \\
S_{2}(r, s) & =\sum_{j=0}^{(N-3) / 2} \zeta(2 j) \zeta(N-2 j) \sum_{i=1}^{r}(-1)^{i}\left(\begin{array}{c}
r+s-i-1 \\
s-1
\end{array}\right)\left(\begin{array}{c}
N-2 j-1 \\
N-i-1
\end{array}\right), \\
S_{3}(r, s) & =\sum_{i=1}^{r}\left(\begin{array}{c}
r+s-i-1 \\
s-1
\end{array}\right) \zeta(0) \zeta(N)=\left(\begin{array}{c}
r+s-1 \\
s
\end{array}\right) \zeta(0) \zeta(N) \\
S_{4}(r, s) & =\sum_{i=3}^{r}\left(\begin{array}{c}
r+s-i-1 \\
s-1
\end{array}\right) \zeta(i) \zeta(N-i) .
\end{aligned}
$$

Using Lemma 2.1 (with $m=r-1, n=s-1, p=q=N-2 j-1$ ), we obtain

$$
\begin{aligned}
S_{1}(r, s)= & \sum_{j=0}^{(N-3) / 2} \zeta(2 j) \zeta(N-2 j) \sum_{i=0}^{r-1}(-1)^{i+1}\left(\begin{array}{c}
r+s-i-2 \\
s-1
\end{array}\right)\left(\begin{array}{c}
N-2 j-1 \\
i
\end{array}\right) \\
= & (-1)^{r} \sum_{j=0}^{(N-3) / 2}\left(\begin{array}{c}
N-2 j-s-1 \\
r-1
\end{array}\right) \zeta(2 j) \zeta(N-2 j) \\
= & (-1)^{r}\left(\sum_{j=0}^{[(N-r-s) / 2]}+\sum_{j=[(N-s+1) / 2]}^{(N-3) / 2}\right)\left(\begin{array}{c}
N-2 j-s-1 \\
r-1
\end{array}\right) \\
& \times \zeta(2 j) \zeta(N-2 j) .
\end{aligned}
$$


Again using Lemma 2.1 (with $m=r, n=s-1, p=N-1, q=N-2 j-1$ ), we obtain

$$
\begin{aligned}
S_{2}(r, s)= & \sum_{j=0}^{(N-3) / 2} \zeta(2 j) \zeta(N-2 j) \sum_{i=0}^{r}(-1)^{i}\left(\begin{array}{c}
r+s-i-1 \\
s-1
\end{array}\right)\left(\begin{array}{c}
N-2 j-1 \\
N-i-1
\end{array}\right) \\
& -\sum_{j=0}^{(N-3) / 2} \zeta(2 j) \zeta(N-2 j)\left(\begin{array}{c}
r+s-1 \\
s-1
\end{array}\right)\left(\begin{array}{c}
N-2 j-1 \\
N-1
\end{array}\right) \\
= & (-1)^{r} \sum_{j=0}^{[r / 2]}\left(\begin{array}{c}
N-2 j-s-1 \\
r-2 j
\end{array}\right) \zeta(2 j) \zeta(N-2 j)-\left(\begin{array}{c}
r+s-1 \\
s-1
\end{array}\right) \zeta(0) \zeta(N) .
\end{aligned}
$$

Next, using (2.1) we have

$$
\begin{aligned}
S_{4}(r, s) & =\sum_{j=[(N-r+1) / 2]}^{(N-3) / 2}\left(\begin{array}{c}
r+s-N+2 j-1 \\
s-1
\end{array}\right) \zeta(2 j) \zeta(N-2 j) \\
& =(-1)^{s-1} \sum_{j=[(N-r+1) / 2]}^{(N-3) / 2}\left(\begin{array}{c}
N-2 j-r-1 \\
s-1
\end{array}\right) \zeta(2 j) \zeta(N-2 j) .
\end{aligned}
$$

Clearly

$$
S_{2}(r, s)+S_{3}(s, r)=(-1)^{r} \sum_{j=0}^{[r / 2]}\left(\begin{array}{c}
N-2 j-s-1 \\
r-2 j
\end{array}\right) \zeta(2 j) \zeta(N-2 j) .
$$

Also,

$$
\begin{aligned}
S_{1}(r, s)+S_{4}(s, r)= & (-1)^{r} \sum_{j=0}^{[(N-r-s) / 2]}\left(\begin{array}{c}
N-2 j-s-1 \\
r-1
\end{array}\right) \zeta(2 j) \zeta(N-2 j) \\
= & (-1)^{r} \sum_{j=0}^{[(N-r-s-1) / 2]}\left(\begin{array}{c}
N-2 j-s-1 \\
r-1
\end{array}\right) \zeta(2 j) \zeta(N-2 j) \\
& +\frac{1}{2}\left\{(-1)^{r}-(-1)^{s}\right\} \zeta(N-r-s) \zeta(r+s),
\end{aligned}
$$

where the last term occurs since $[(N-r-s) / 2]=[(N-r-s-1) / 2]+1$ when $r+s$ is odd. Now by (1.13) we have

$$
\begin{aligned}
E_{N}(r, s)= & S_{1}(r, s)+S_{4}(s, r)+S_{2}(r, s)+S_{3}(s, r) \\
& -\frac{1}{2}\left\{(-1)^{r}-(-1)^{s}\right\} \zeta(N-r-s) \zeta(r+s) .
\end{aligned}
$$


Hence

$$
\begin{aligned}
E_{N}(s, r)= & S_{1}(s, r)+S_{4}(r, s)+S_{2}(s, r)+S_{3}(r, s) \\
& -\frac{1}{2}\left\{(-1)^{s}-(-1)^{r}\right\} \zeta(N-r-s) \zeta(r+s)
\end{aligned}
$$

so that

$$
E_{N}(r, s)+E_{N}(s, r)=U_{N}(r, s)+U_{N}(s, r)=T(r, s, N-r-s) .
$$

5. Conclusion. It appears to be a difficult open problem to determine an explicit formula for $T(r, s, t)$ when $r+s+t=N$ is even. Such a formula is known for a general even $N$ in the following cases (where we have taken $r \geq s$ in view of (1.2)):

\begin{tabular}{cccl}
\hline$r$ & $s$ & $t$ & \multicolumn{1}{c}{ Reference } \\
\hline 0 & 0 & $N$ & {$[6$, Theorem 5$]$} \\
$r$ & $N-r$ & 0 & {$[6$, eq. $(6)]$} \\
1 & 1 & $N-2$ & {$[6$, eq. $(8)]$} \\
1 & 0 & $N-1$ & {$[6$, eq. (10)] } \\
$r$ & $N-r-1$ & 1 & {$[6$, Theorem 3$]$} \\
$N / 2$ & 0 & $N / 2$ & {$[6$, Theorem 6$]$} \\
$N / 3$ & $N / 3$ & $N / 3$ & {$[5$, p. 254] } \\
$N / 3$ & $N / 3-1$ & $N / 3+1$ & (by preceding result and $(1.5))$ \\
\hline
\end{tabular}

In each of these cases except the first (see (1.10)) the formula for $T(r, s, t)$ can be given as a linear combination of $\zeta(N)$ and $\zeta(i) \zeta(N-i), i=2,3, \ldots, N / 2$. It would be interesting to know if this is the case in general.

Acknowledgements. The first and third authors would like to acknowledge the hospitality of the Centre for Research in Algebra and Number Theory during their stays at Carleton University.

\section{References}

[1] H. W. Gould, Combinatorial Identities, Morgantown, W.Va., 1972.

[2] L. J. Mordell, On the evaluation of some multiple series, J. London Math. Soc. 33 (1958), 368-371.

[3] N. Nielsen, Die Gammafunktion, Chelsea, Bronx, 1965.

[4] J. Riordan, Combinatorial Identities, Wiley, New York, 1968. 
[5] M. V. Subbarao and R. Sitaramachandrarao, On some infinite series of L. J. Mordell and their analogues, Pacific J. Math. 119 (1985), 245-255.

[6] L. Tornheim, Harmonic double series, Amer. J. Math. 72 (1950), 303-314.

Department of Mathematics

Canisius College

Buffalo, New York 14208

U.S.A.

Centre for Research in Algebra and Number Theory

Department of Mathematics and Statistics

Carleton University

Ottawa, Ontario, Canada K1S 5B6

Information Department

The People's University of China

Beijing, People's Republic of China 100872

Received on 16.5.1995

and in revised form on 4.8 .1995 